$08,12,18$

\title{
Рассеяние электронов дефектами малого радиуса и сопротивление графена
}

\author{
(C) Н.Е. Фирсова ${ }^{1,2}$, С.А. Ктиторов ${ }^{1,3,9}$ \\ ${ }^{1}$ Физико-технический институт им. А.Ф. Иофрфе РАН, \\ Санкт-Петербург, Россия \\ ${ }^{2}$ Санкт-Петербургский политехнический университет им. Петра Великого, \\ Санкт-Петербург, Россия \\ ${ }^{3}$ Санкт-Петербургский электротехнический университет им. В.И. Ульянова (Ленина), \\ Санкт-Петербург, Россия \\ I E-mail: ktitorov@mail.ioffe.ru
}

(Поступила в Редакцию 26 ноября 2018 г.

В окончательной редакции 26 ноября 2018 г.

Принята к публикации 28 ноября 2018 г.)

\begin{abstract}
Рассмотрено рассеяние электронов короткодействующими дефектами в плоском монослойном графене. Использована аппроксимация этого взаимодействия дельтаобразным потенциалом, сосредоточенным на окружности малого радиуса, что обеспечивает подавление нефизических коротковолновых мод. Проанализирован вклад этого рассеяния в сопротивление графена. Полученные результаты находятся в хорошем согласии с экспериментом на подвешенном отожженном монослойном графене. Это дает возможность определения параметров аппроксимирующего потенциала на основе экспериментальных данных о сопротивлении графена, что важно для приложений.
\end{abstract}

DOI: 10.21883/FTT.2019.04.47421.328

\section{1. Введение}

Уникальные свойства графена привлекают внимание исследователей с момента открытия благодаря специфическим особенностям его электронной и фононной подсистем и перспективным приложениям. Бесщелевой квазирелятивистский электронный спектр позволил построить теорию проводимости, основанную на релятивистской теории рассеяния [1].

В настоящей работе мы анализируем влияние короткодействующих дефектов на сопротивление (здесь и далее: удельное сопротивление) плоского монослойного графена. Мы используем теорию рассеяния для двумерного уравнения Дирака, развитую Новиковым [2] и борновское приближение. Короткодействующие дефекты моделируются предложенным в $[3,4]$ потенциалом типа „дельта функция на окружности“, что обеспечивает подавление нефизических коротковолновых возбуждений. Цель данной работы состоит в теоретическом исследовании сопротивления плоского монослойного графена благодаря рассеянию электронов точечными дефектами. Вблизи дираковской точки мы используем полученную в [5] методом парциальных волн формулу для проводимости. Кроме того производится вычисление сопротивления и проводимости в широком диапазоне энергий в борновском приближении. Мы сравниваем наши теоретические результаты с известными экспериментальными данными, полученными на свободно подвешенном монослойном графене [6].
Уравнение Дирака, описывающее электронные состояния в графене, имеют вид [1]:

$$
\hat{H} \psi(x, y) \equiv\left[-i \hbar v_{F} \sum_{\mu=1}^{2} \hat{\sigma}_{\mu} \delta_{x_{\mu}}+\hat{V}\right] \psi(x, y)=E \psi(x, y) \text {, }
$$

где $v_{F}$ - скорость Ферми, $\sigma_{\mu}$ - матрицы Паули, $\psi(r)-$ двухкомпонентный спинор. Мы используем потенциал типа „дельта-функция на окружности“, что обеспечивает подавление нефизических коротковолновых возбуждений $[3,4]$

$$
V(r)=-V_{0} \delta\left(r-r_{0}\right)
$$

Предполагается, что возмущение локально сдвигает точку Дирака по оси энергий. Введем безразмерные переменные

$$
\begin{gathered}
\hat{H}=\frac{\hat{H}}{\hbar v_{F} / r_{0}}, E=\frac{E}{\hbar v_{F} / r_{0}}, V_{0}=\frac{V_{0}}{\hbar v_{F}}, \\
x=x / r_{0}, \quad y=y / r_{0}, \quad r=r / r_{0}, k=k r_{0} .
\end{gathered}
$$

Безразмерный потенциал принимает вид

$$
V(r)=-V_{0} \delta(r-1) .
$$

Уравнение (1) можно переписать

$$
\hat{H} \psi(x, y) \equiv\left[-i \sum_{\mu=1}^{2} \hat{\sigma}_{\mu} \delta_{x_{\mu}}+\hat{V}\right] \psi(x, y)=E \psi(x, y) .
$$




\section{2. Матрица рассеяния: представление парциальных волн}

Вычислив отношение амплитуд сходящихся и расходящихся волн, мы получаем компоненты S-матрицы в представлении парциальных волн [4]

$$
S_{j}(k)=-\frac{F_{j}^{(2)}(k)}{F_{j}^{(1)}(k)}, j= \pm 1 / 2, \pm 3 / 2, k=E,
$$

где

$$
\begin{gathered}
F_{j}^{(\alpha)}=\left[I_{j-1 / 2}(k) H_{j+1 / 2}^{(\alpha)}(k)-I_{j+1 / 2}(k) H_{j-1 / 2}^{(\alpha)}(k)\right] . \\
\tan V_{0}\left[I_{j+1 / 2}(k) H_{j+1 / 2}^{(\alpha)}(k)+I_{j-1 / 2}(k) H_{j-1 / 2}^{(\alpha)}(k)\right], \\
\alpha=1,2 .
\end{gathered}
$$

В результате получаем асимптотически точное в пределе $k \rightarrow 0$ выражение для транспортного времени релаксации [5]

$$
\tau_{t r}^{-1}(k)=\pi^{2} k v_{F} N_{i} \tan ^{2} V_{0},
$$

где $N_{i}=r_{0}^{2} N_{i}, N_{i}-$ концентрация дефектов. Предположив справедливость уравнения Больцмана, мы получаем следующую формулу для проводимости при низкой температуре $k_{B} T \ll E_{F}$ :

$$
\sigma\left(E_{F}\right)=\sigma_{0} \frac{E_{F} \tau_{t r}\left(E_{F}\right)}{\hbar}, \tilde{\sigma}=\sigma / \sigma_{0},
$$

где $\sigma_{0}=\frac{4 e^{2}}{h}$; множитель 4 учитывает спиновое и орбитальное вырождение, $h=2 \pi \hbar-$ постоянная Планка. Транспортное время релаксации $\tau_{t r}\left(E_{F}\right)$ и проводимость $\sigma\left(E_{F}\right)$ были вычислены в [5]. асимптотически точно в пределе низкой энергии Ферми. Следовательно, больцмановское сопротивление $\rho$ имеет вид [5]

$$
\begin{gathered}
\rho=\sigma^{-1}=\sigma_{0}^{-1} \pi^{2} N_{i} \cdot \tan ^{2} V_{0}, \\
\tilde{\rho}=\rho / \rho_{0}, \quad \rho_{0}=\sigma_{0}^{-1},
\end{gathered}
$$

где $\tilde{\rho}-$ безразмерное сопротивление. Мы представляем формулу для сопротивления, справедливую в пределе длинных волн и произвольной величине возмущения в пределах действия уравнения Больцмана

$$
E_{F} \tau_{t r} / \hbar>1
$$

Далее мы вычислим сопротивление в широком диапазоне дебройлевских длин волн волн, но в пределе малой величины потенциала дефекта. Для этого мы применим борновское приближение [2].

\section{3. Борновское приближение}

Уравнение Дирака (1) может быть переписано в интегральной форме [2]

$$
\begin{aligned}
\psi_{\text {scat }}= & -\int d x^{\prime} d y^{\prime} G\left(x-x^{\prime}, y-y^{\prime}\right) \\
& \times\left[i \hat{\sigma}_{\mu} \delta_{x_{\mu}}+E\right] \hat{V}\left(x^{\prime}, y^{\prime}\right) u_{p} e^{i p r^{\prime}},
\end{aligned}
$$

где функция Грина имеет вид

$$
\begin{aligned}
G(\mathbf{r}) & =\int \frac{d k_{x} d k_{y}}{(2 \pi)^{2}} \frac{e^{i\left(k_{x} x+k_{y} y\right)}}{k^{2}-(E+i 0 \operatorname{sgn} E)^{2}} \\
& =\frac{i \pi}{4 \pi} \operatorname{sgn} E H_{0}^{(1)}(k r), \\
H_{m}^{(1,2)} & =J_{m}(x) \pm i N_{m}(x), \quad r=\sqrt{x^{2}+y^{2}} .
\end{aligned}
$$

Уравнение (11) представлено в первом борновском приближении. Решение можно записать в следующем виде:

$$
\psi=u_{k x} e^{i k x}+\frac{f(\theta)}{\sqrt{-i r}} u_{p \theta} e^{i k r},
$$

где амплитуда рассеяния имеет вид

$$
f^{\text {Born }}(k, \theta)=-\sqrt{k / 8 \pi} V_{q}\left(1+e^{i \theta}\right),
$$

причем

$$
\theta=\angle\left(\mathbf{k}^{\prime}, \mathbf{k}\right), \quad q=2 \kappa \sin (\theta / 2) .
$$

Преобразование Фурье имеет вид

$$
V_{q}=\int d x d y e^{-i\left(q_{x} x+q_{y} y\right)} V(r) .
$$

Введем обозначение: $\tilde{\Sigma}_{t r}=\Sigma_{t r} / r_{0}$, где $\Sigma_{t r}-$ транспортное сечение. Подставив (14) в (13) и используя известную формулу для транспортного сечения [2]

$$
\tilde{\Sigma}_{t r}=\int d \theta(1-\cos \theta)|f(\theta)|^{2},
$$

мы получаем безразмерное транспортное сечение для нашей задачи в следующем виде:

$$
\tilde{\Sigma}_{t r}^{\text {Born }}(k)=\pi k V_{0}^{2} I(k) .
$$

Здесь функция $I(k)$ определена формулой

$$
I(k)=\int_{0}^{2 \pi} d \theta \sin ^{2}(\theta) J_{0}^{2}[2 k \sin (\theta / 2)] .
$$

Этот интеграл может быть выражен через гипергеометрическую функцию [7]

$$
\begin{aligned}
I(k)= & \frac{1}{2} \Gamma\left[\begin{array}{c}
3 / 2,1 / 2 \\
1,1,2
\end{array}\right] \\
& \times{ }_{4} F_{5}\left(3 / 2,1 / 2,1 / 2,1 ; 1,1,1,3 / 2 ;-k^{2}\right) .
\end{aligned}
$$


Здесь

$$
\Gamma\left[\begin{array}{c}
\alpha_{1}, \alpha_{2} \\
\beta_{1}, \beta_{2}, \beta_{3}
\end{array}\right]=\frac{\Gamma\left(\alpha_{1}\right) \Gamma\left(\alpha_{2}\right)}{\Gamma\left(\beta_{1}\right) \Gamma\left(\beta_{2}\right) \Gamma\left(\beta_{3}\right)},
$$

$\Gamma(\alpha)$ - гамма функция, ${ }_{4} F_{5}$ - обобщенная гипергеометрическая функция

$$
\begin{aligned}
{ }_{4} F_{5}\left(\alpha_{1}, \alpha_{2}, \alpha_{3}, \alpha_{4} ; \beta_{1}, \beta_{2}, \beta_{3}, \beta_{4}, \beta_{5} ; z\right) \\
\quad=\sum_{n=0}^{\infty} \frac{\left(\alpha_{1}\right)_{n}\left(\alpha_{2}\right)_{n}\left(\alpha_{3}\right)_{n}\left(\alpha_{4}\right)_{n}}{\left(\beta_{1}\right)_{n}\left(\beta_{2}\right)_{n}\left(\beta_{3}\right)_{n}\left(\beta_{4}\right)_{n}\left(\beta_{5}\right)_{n}} \frac{z^{n}}{n !},
\end{aligned}
$$

где $\left(\alpha_{1}\right)_{n}=\frac{\Gamma(\alpha+n)}{\Gamma(n)}-$ повышающий символ Похгаммера.

Используя (15) мы находим время релаксации

$$
\begin{aligned}
\frac{1}{\tau_{t r}^{\text {Born }}} & =\Sigma_{t r}^{\text {Born }} N_{i} v_{F}=\tilde{\Sigma}_{t r}^{\text {Born }} r_{0} N_{i} v_{F} \\
& =\pi k_{i} V_{0}^{2} r_{0} N_{i} v_{F} I(k)=\pi N_{i} V_{0}^{2} k_{F} v_{F} I(k) .
\end{aligned}
$$

Подставив (19) в (8), (9), получаем формулу для безразмерного сопротивления

$$
\tilde{\rho}^{\text {Born }}=\pi N_{i} V_{0}^{2} I(k) .
$$

\section{4. Соотношение между данными рассеяния электронов и сопротивлением}

Нетрудно убедиться, что $I(k) \rightarrow \pi$ в пределе $k \rightarrow 0$. Таким образом, выражения (9) и (20) для безразмерного сопротивления асимптотически идентичны в пределе $V_{0} \rightarrow 0, k \rightarrow 0$, т.е. в окрестности максимума сопротивления. Численное вычисление функций (9) и (20) для сопротивления позволяет сравнить теоретическую кривую (рис. 1,2) и измеренную в эксперименте на свободно подвешенном графене [6] (рис. 3,4) кривую сопротивления в окрестности дираковской точки. Сравнение показывает близкое подобие этих кривых при

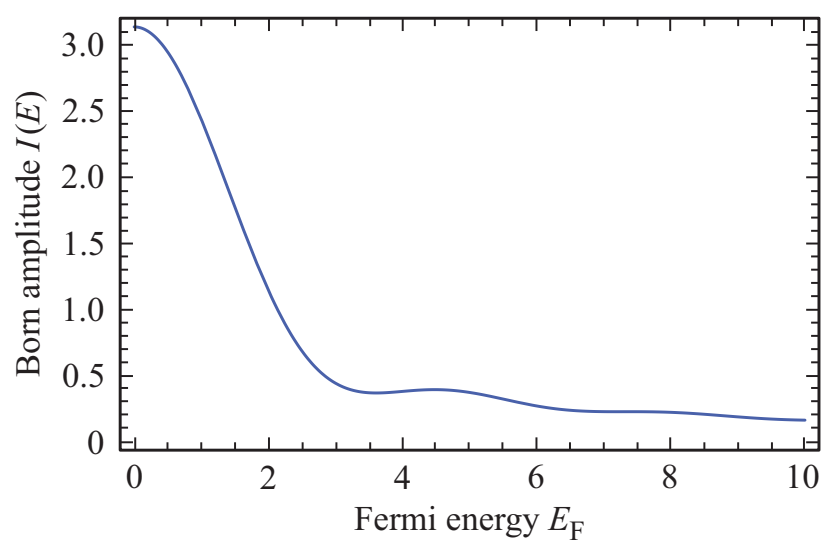

Рис. 1. Борновская амплитуда рассеяния как функция энергии Ферми (безразмерные переменные).

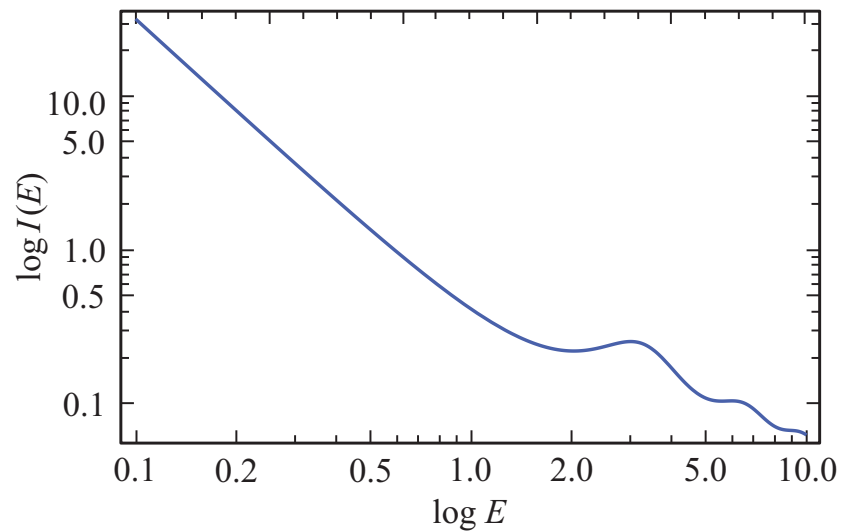

Рис. 2. Лог - лог график для борновской амплитуды рассеяния как функции энергии Ферми.

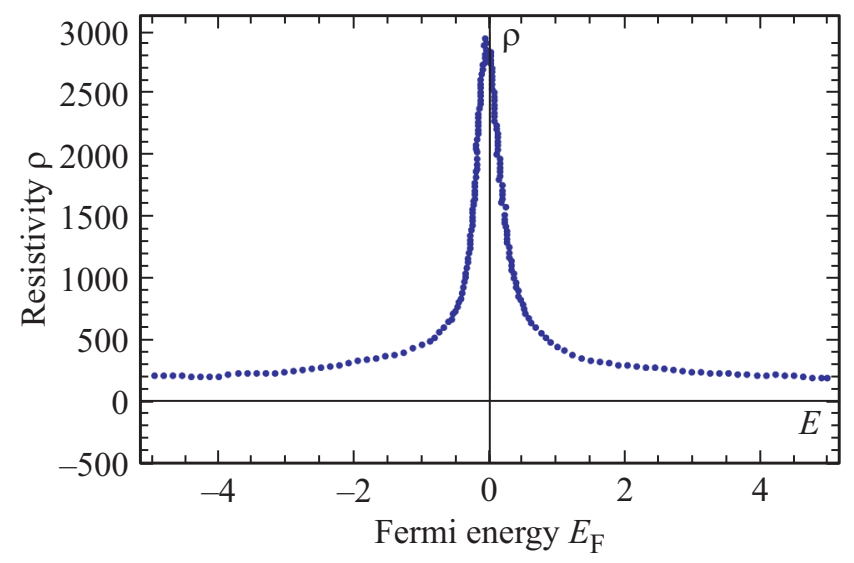

Рис. 3. Измеренное сопротивление как функция энергии Ферми.

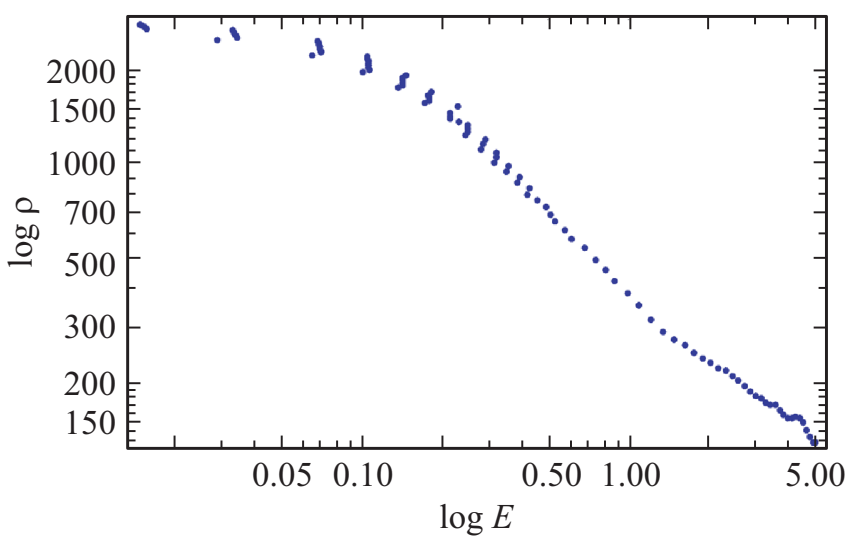

Рис. 4. Лог - лог график для измеренного сопротивления как функции энергии Ферми.

малых энергиях Ферми, что указывает на возможность установления полезных соотношений между данными рассеяния и сопротивлением. Имеются два диапазона энергий Ферми, где сравнение с экспериментальными данными может быть эффективно проведено: $(a)-$ в 
окрестноси максимума сопротивления и $(b)-$ на склоне контура сопротивления. Так как наша теория учитывает только рассеяние на нейтральных примесях, мы сравниваем ее результаты с экпериментом на отожженном подвешенном графене, содержащем минимальное количество заряженных дефектов [6]. Форма кривой $\rho\left(E_{F}\right)$, полученная в [6] хорошо описывается функцией $I\left(E_{F}\right)$ (см. рис. 1). Степенное поведение $\rho\left(E_{F}\right)$ на боковом склоне универсально и следует из короткодействующего характера взаимодествия электронов с дефектами. Лог - лог график этой фунеции, представленный на рис. 2, дает с хорошей точностью показатель степени, равный -1 . Заметим, что экспериментальная кривая (рис. 3,4$)$ начинает отклоняться от закона $1 / E_{F}$ при меньшей величине $E_{F}$, что можно объяснить присутствием иных дефектов. Напротив, поведение сопротивения в окрестности максимума не универсально и может меняться от образца к образцу. Одной из причин является дираковская сингулярность. Различные подходы дают разные величины максимально теоретически возможного сопротивления $[8,9]$. Например в [8] результат не зависит от данных рассеяния, поскольку сопротивление имеет дифракционную природу. Максимально возможное сопротивление равно в этом случае

$$
\frac{h}{4 e^{2}} \eta^{-1}
$$

где $\eta$ - численный множитель, его величина определяется порядком предельных переходов $\omega \rightarrow 0, E_{F} \rightarrow 0$, $T \rightarrow 0$. Согласно [8], хорошее согласие с экспериментом достигается при $\eta=\pi / 4$. Тогда максимальное значение сопротивления $\rho_{\text {trans }}^{(1)}$ (обозначение Циглера [8]) принимает вид:

$$
\rho_{\max }^{(1)}=\frac{h}{\pi e^{2}}=\frac{24}{\pi} \mathrm{k} \Omega \approx 7.64 \mathrm{k} \Omega
$$

Минимальная оптическая проводимость получена в [9] на основе решения уравнения фон Неймана для матрицы плотности. Показано в [9], что $\rho_{\max }^{\text {opt }}=\frac{h}{\pi^{2} e^{2}}$.

В отличие от работ $[8,9]$, наша формула основана на данных рассеяния. Мы оцениваем минимальную проводимость из условия справедливсти уравнения Больцмана. Мы вводим квазиклассически определенную величину $\rho_{\max }^{q c}$ для максимально возможного сопротивления основываясь на аргументации Нэвила Мотта, согласно которой длина пробега не может быть меньше, чем длина волны электрона. Следовательно $\rho_{\max }^{q c}$ определено условием

$$
E_{f} \tau_{t r} \hbar=k_{F} l>1,
$$

где $l$ - длина пробега. Получаем следующую оценку для $\rho_{\text {max }}^{q c}$ :

$$
\rho_{\max }^{q c}=h / 4 e^{2} \approx 6 \mathrm{k} \Omega .
$$

Сравнивая с результатом измерений на подвешенном графене, проведенных в работе [6], получаем $\rho_{\max }^{\exp } \approx 2.4 \mathrm{k} \Omega$, т.е. в 2.5 раза меньше. Это указывает на важность квантовых эффектов вблизи максимума сопротивления.

Введем меру интенсивности рассеяния $P$

$$
P=N_{i} r_{0}^{2} \tan ^{2} \frac{V_{0}}{\hbar v_{F}}=N_{i} \tan ^{2} V_{0}
$$

В случае слабого рассеяния $V_{0}=\frac{V_{0}}{\hbar v_{F}} \ll 1$ мы имеем

$$
P=N_{i}^{2} r_{0}^{2}\left(\frac{V_{0}}{\hbar v_{F}}\right)^{2}=N_{i} V_{0}^{2}
$$

Формулы (9), (20) при $V_{0} \ll \hbar v_{F}$ принимают вид

$$
P=\tilde{\rho}_{\max } / \pi^{2} .
$$

Полученная формула для $\tilde{\rho}_{\max }$ может быть переписана в размерном виде

$$
\rho_{\max }=\frac{h}{4 e^{2}} \pi^{2} P=\frac{h}{4 e^{2}} N_{i} r_{0}^{2} \tan ^{2} \frac{V_{0}}{\hbar v_{F}}
$$

или для $V_{0} \ll \hbar v_{F}$ :

$$
\rho_{\max } \approx \frac{h}{4 e^{2}} N_{i} r_{0}^{2}\left(\frac{V_{0}}{\hbar v_{F}}\right)^{2} .
$$

Формулы (28), (29) дают возможность получения информации о параметрах дефектов (например, о параметре интенсивности рассеяния $P$ ) основываясь на таких экспериментальных данных как сопротивление при малой энергии Ферми.

\section{5. Заключение}

В настоящей работе мы исследовали рассеяние электронов в монослойном графене на короткодействующих дефектах и его влияние на сопротивление. Рассмотрение проводилось в рамках плоской модели графена с потенциалом типа дельта - функция на окружности малого радиуса, аппроксимирующим короткодействующие дефекты. Эта модель короткодействующего дефекта обеспечивает регуляризацию задачи рассеяния подавляя нефизические коротковолновые возбуждения. В низкоэнергетическом пределе мы используем зависимость проводимости от энергии Ферми, полученную в [5] методом парциальных волн. Для этого в [5] было решено соответствующее $2+1$ - мерное уравнение Дирака и асимптотический анализ полученной $S$ - матрицы позволил получить используемую нами формулу для проводимости.

Случай относительно высоких энергий мы рассматриваем используя борновское приближение. Численные вычисления были проведены в широком диапаоне электронных энергий $E$. Показано, что сопротивление имеет максимум при $E=0$ и убывает как $1 / E$ вплоть до не слишком больших энергий, при которых уравнение Дирака с модельным потенциалом перестает работать (см. рис. 1,2). 
Мы сравнили наши теоретические кривые (рис. 1,2) и измерения на отожженном подвешенном монослойном графене, выполненные в [6] (рис. 3,4). Из этого сравнения сделан вывод, что использованный нами модельный потенциал удовлетворительно описывает сопротивление подвешенного графена благодаря минимизации концентрации заряженных примесей в результате отжига. Результаты нашей теории находятся в хорошем согласии с экспериментом на подвешенном монослойном графене вплоть до умеренно низких энергий Ферми.

Сравнивая наши теоретические результаты (рис. 1,2) с экспериментальными данными (рис. 3,4) приходим к выводу, что экспериментальная кривая может быть аппроксимирована функцией $1 / E$ при меньших энергиях, чем это предсказывает наша теория в результате действия неучтенных механизмов рассеяния.

Наша теория, примененная к анализу экспериментальных данных, полученных на подвешенном монослойном графене дает инструмент для оценки интенсивности рассеяния по измеренному сопротивлению при малых энергиях Ферми.

Полученные результаты важны для приложений. Одно из важнейших - создание полевого транзистора на графене. Колоссальная электронная подвижность монослойного графена делает его перспективным материалом для высокочастотных приборов.

\section{Список литературы}

[1] K.S. Novoselov, A.K. Geim, S.V. Morozov, D. Jiang, M.I. Katsnelson, I.V. Grigorieva, A.A.Firsov. Nature 438, 197 (2005).

[2] D.S. Novikov. Phys. Rev. B 76, 245 (2007).

[3] N.E. Firsova, S.A. Ktitorov, P. Pogorelov. Phys. Lett. A 373, 525 (2009).

[4] N.E. Firsova, S.A. Ktitorov. Phys. Lett. A 374, 1270 (2010).

[5] N.E. Firsova. Nanosystems: Physics, Chemistry, Mathematics 4, 538 (2013).

[6] K.I. Bolotin, K.I. Sikesh, Z. Jiang, M. Klimac, G. Fudenberga, J. Honec, P. Kima, H.L. Storme. Solid State Commun. 146, 351 (2008).

[7] M. Abramowitz, I. Stegun. Handbook of Mathematical Functions with Formulas, Graphs and Mathematical Tables. National Bureau of Standards (1972).

[8] K. Ziegler. Phys. Rev. B 75, 233407 (2007).

[9] N.E. Firsova. Photonics and Nanostructure - Fundamentals and Applications 26, 8 (2017).

Редактор Т.Н. Василевская 